EPJ Web of Conferences 49, 17003 (2013)

DOI: $10.1051 /$ epjconf/20134917003

(C) Owned by the authors, published by EDP Sciences, 2013

\title{
Top pair production at CMS (includes charge asymmetry and ttbar resonance)
}

\author{
Benjamin Klein 1 , a, b \\ ${ }^{1}$ Ghent University, Department of Physics and Astronomy, Proeftuinstraat 86, B-9000 Ghent (Belgium) \\ E-mail: benjamin.klein@cern.ch
}

\begin{abstract}
.
Measurements of top quark pair production are presented based on data recorded with the Compact Muon Solenoid (CMS) detector at the Large Hadron Collider (LHC) in 2011 and 2012. Inclusive and differential top quark pair production cross section measurements in various channels are described. Inclusive as well as differential measurements of the charge asymmetry in top quark pair events are summarised and a method for the extraction of the strong coupling $\alpha_{s}$ from the measured top pair production cross section is discussed. All measurements are in agreement with the Standard Model predictions.
\end{abstract}

\section{Introduction}

The top quark is the heaviest fundamental particle described by the Standard Model of particle physics. Due to the high mass its lifetime is shorter than the hadronisation time. This makes it possible to study the properties of top quarks based on their decay products. Top quark production is an important process to perform precision tests of the Standard Model. Furthermore, in many predicted processes of physics beyond the Standard Model top quark events are one of the dominant background contributions.

At the LHC top quarks are produced with a rate more than 20 times higher than in any previous particle physics experiment and the LHC is often referred to as a top quark factory. The CMS detector [1] is one of the two large multi-purpose detectors at the LHC and allows for a study of a wide range of physics processes. In 2011 data, an integrated luminosity of $5 \mathrm{fb}^{-1}$ at a centre-of-mass energy of $\sqrt{s}=7 \mathrm{TeV}$ is available for physics studies and in 2012 an integrated luminosity of $22 \mathrm{fb}^{-1}$ at $\sqrt{s}=8 \mathrm{TeV}$ has been recorded.

\section{Inclusive Cross Section Measurements}

Measurements of the inclusive top quark pair production cross section are available in various channels. At a centre-of-mass energy of $\sqrt{s}=7 \mathrm{TeV}$ measurements have been performed in the semi-leptonic channels with electrons, muons and tau leptons, in the di-leptonic channels measurements are available in the di-electron, di-muon, electron-muon, electron-tau and muon-tau channels. Also a measurement in the all hadronic channel has been performed. A summary of the measurements is presented in Figure 1. The most precise individual measurement at

a. Speaker.

b. On behalf of the CMS collaboration.

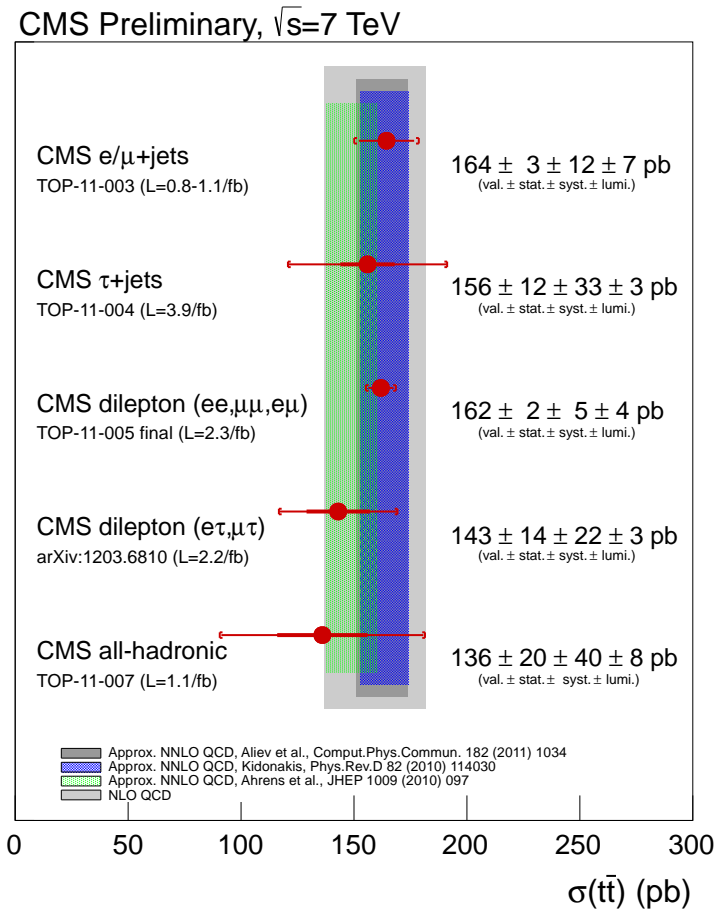

Figure 1. Top pair cross section summary plot for $\sqrt{s}=7 \mathrm{TeV}$.

$\sqrt{s}=7 \mathrm{TeV}$ is the measurement in the di-leptonic ee, $\mu \mu$ and $e \mu$ channels and yields as result

$$
\sigma_{t \bar{t}}=162 \pm 2 \text { (stat.) } \pm 5 \text { (syst.) } \pm 4 \text { (lumi) pb. }
$$

All results presented in Figure 1 are in agreement with each other and with the Standard Model predictions.

At a centre-of-mass energy of $\sqrt{s}=8 \mathrm{TeV}$ measurements in the di-leptonic channels and the semi-leptonic channels with electrons and muons are available. 


\subsection{Measurement in the di-leptonic channel at} $\sqrt{s}=8 \mathrm{TeV}$

Events are selected in the di-leptonic channels $e e, \mu \mu$ and $e \mu$. A counting method is used to measure the cross section. The event signature of two charged leptons and two jets, with at least one jet identified as originating from a $b$-quark, guarantees a high purity of the selected event sample and makes a precise determination of the cross section possible. The dominant backgrounds are Drell-Yan $Z / \gamma^{*} \rightarrow \ell \ell$ (with $\ell=\mu, e$ ) events in the $e e$ and $\mu \mu$ channels and $Z / \gamma^{*} \rightarrow \tau \tau \rightarrow\left(e v_{e} v_{\tau}\right)\left(\mu v_{\mu} v_{\tau}\right)$ events in the $e \mu$ channel. Significant sources of systematic uncertainties are the uncertainty on the jet energy scale and resolution and the measurement of lepton efficiencies.

A combination of the three decay channels with the BLUE method yields

$$
\sigma_{t \bar{t}}=227 \pm 3 \text { (stat.) } \pm 11 \text { (syst.) } \pm 10 \text { (lumi) pb, }
$$

which is in good agreement with the Standard Model expectation of $\sigma(\sqrt{s}=8 \mathrm{TeV})=225.2 \mathrm{pb}$ [3].

The cross section ratio for a centre-of-mass energy of $\sqrt{s}=8 \mathrm{TeV}$ and $\sqrt{s}=7 \mathrm{TeV}$ results in

$$
\sigma(8 \mathrm{TeV}) / \sigma(7 \mathrm{TeV})=1.41 \pm 0.11 \text {. }
$$

\subsection{Measurement in the semi-leptonic channel at $\sqrt{s}=8 \mathrm{TeV}$}

A measurement in the semi-leptonic channels with an electron or muon is presented. A simultaneous template fit is performed on the invariant mass distribution of the three jet system in the event with the highest transverse momentum. The results are compatible with the precision measurement described in Section 2.1. The dominant systematic uncertainties are the uncertainty on the identification of $b$-jets and the jet energy scale. The simultaneously measured cross section in the electron and muon channels is

$$
\left.\sigma_{t \bar{t}}=228 \pm 4 \text { (stat. }\right)_{-26}^{+29} \text { (syst.) } \pm 10 \text { (lumi) pb. }
$$

The combination of the measurements in the dileptonic and semi-leptonic channels yields

$$
\sigma_{t \bar{t}}=227 \pm 3 \text { (stat.) } \pm 11 \text { (syst.) } \pm 10 \text { (lumi) pb. }
$$

\section{Differential Cross Section Measurements}

Differential measurements allow precise tests of the Standard Model, are used to tune Standard Model parameters and are sensitive to possible physics beyond the Standard Model.

\subsection{Differential Cross Section as a Function of Kinematic Properties}

The normalised differential cross section is measured in the semi- and di-leptonic final states with electrons and

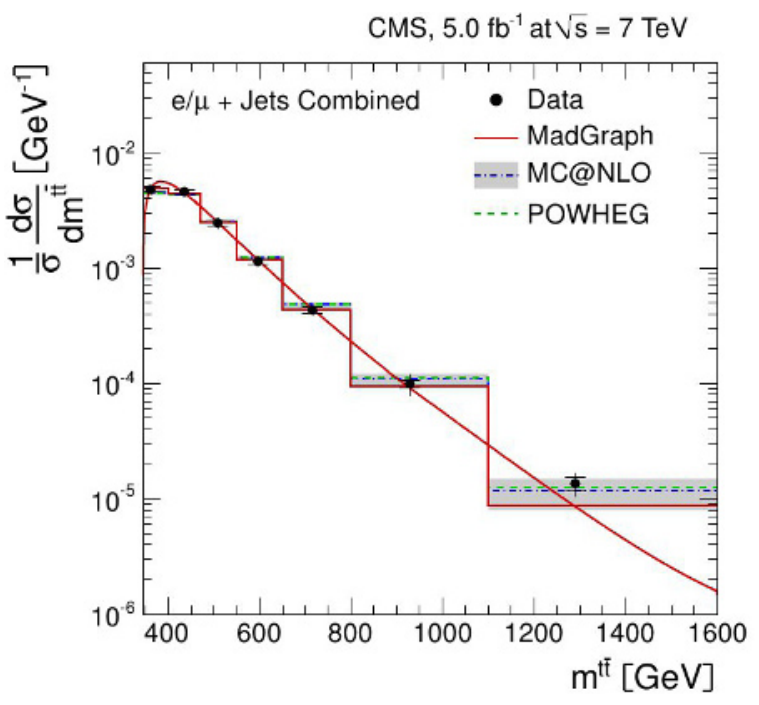

Figure 2. Differential cross section as a function of the invariant mass of the top quark pair system.

muons. The measurement is performed as a counting experiment. The dominant systematic uncertainties are the uncertainty on the background estimation in the semileptonic channels and the uncertainty on trigger and lepton efficiencies in the di-leptonic channels. Migration of events across bin boundaries is corrected by using a regularised unfolding method. Figure 2 shows the differential cross section as a function of the invariant mass of the top quark pair system $m^{t \bar{t}}[10]$ in the semi-leptonic channels. A good agreement between the measurement and the Standard Model prediction is observed up to highest values of $m^{t \bar{t}}$.

With searches for resonances decaying into top quark pairs [11] exclusion limits are set at $95 \%$ c.l. for top-colour $Z^{\prime}$ bosons with narrow (wide) width up to $1.49(2.04) \mathrm{TeV}$ and for Kaluza-Klein excitations up to $1.82 \mathrm{TeV}$.

\section{$3.2 t \bar{t}+E_{T}^{\text {miss }}$}

In many predicted processes of physics beyond the Standard Model, a $t \bar{t}$ decay signature is produced in association with large amounts of missing tansverse energy $E_{T}^{\text {miss }}$. A differential measurement of the top pair cross section as a function of the reconstructed $E_{T}^{\text {miss }}$ in the event [12] is performed in the semi-leptonic $e / \mu+$ jets channels on collision data at $\sqrt{s}=7 \mathrm{TeV}$. In different bins of $E_{T}^{\text {miss }}$ a template fit is performed on the absolute lepton pseudorapidity distribution $\left|\eta_{\mu}\right|$ and $\left|\eta_{e}\right|$. Within the uncertainties a good agreement with the Standard Model prediction is observed in the entire considered $E_{T}^{\text {miss }}$ region as shown in Figure 3.

The dominant systematic uncertainties on the measurement are the uncertainty on the measurement of the missing transverse energy and the theoretical uncertainties due to the choice of factorisation scale and jet-parton matching. 


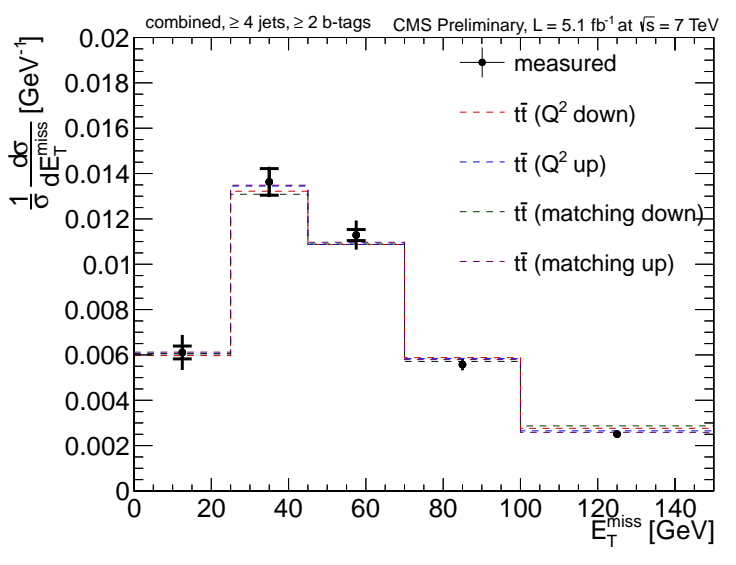

Figure 3. Cross section as a function of the missing transverse energy in top quark pair events within theoretical factorisation scale and jet-parton matching uncertainties.

\section{3 $t \bar{t}+$ jets}

Measurements of the top quark pair cross section as a function of the number of jets in the event are used as a precision test of the performance of Monte Carlo generators. The measurements are sensitive to the factorisation scale and jet-parton matching parameters and can be used to tune those parameters for measurements of other physics processes. A measurement as a function of the number of jets with a transverse momentum larger than $60 \mathrm{GeV}$ is performed using a counting method in the dileptonic channels [13] with electrons and muons. Data from the 2011 data taking period is considered at a centreof-mass energy of $\sqrt{s}=7 \mathrm{TeV}$. Figure 4 compares the measured cross section and the predictions by the MadGraph, MC@NLO and POWHEG Monte Carlo generators. A good agreement is observed between the MadGraph and POWHEG prediction while the MC@NLO prediction shows a steeper spectrum with increasing number of jets. The data is compatible with all Monte Carlo generator predictions within the uncertainties. The dominant systematic uncertainty is the uncertainty due to the jet energy scale.

Figure 5 shows the measured cross section in comparison to the MadGraph generator prediction and the uncertainties on the factorisation scale and jet-parton matching assumptions that were made at production stage. The measured cross section is not sensitive enough to constrain these two parameters. Future measurements with improved uncertainties are necessary to use this kind of information to constrain the factorisation scale and jetparton matching parameters at generator level.

\section{Cross Section Ratio $\sigma(t \bar{t} b \bar{b}) / \sigma(t \bar{t} j j)$}

The channel $t \bar{t} H$ with the decay of the Higgs boson into two bottom quarks $H \rightarrow b b$ is an important channel to measure the coupling of the Higgs boson to the top quark. Higher order QCD corrections predict an irreducible background of $t \bar{t}$ in association with two $b$-quarks

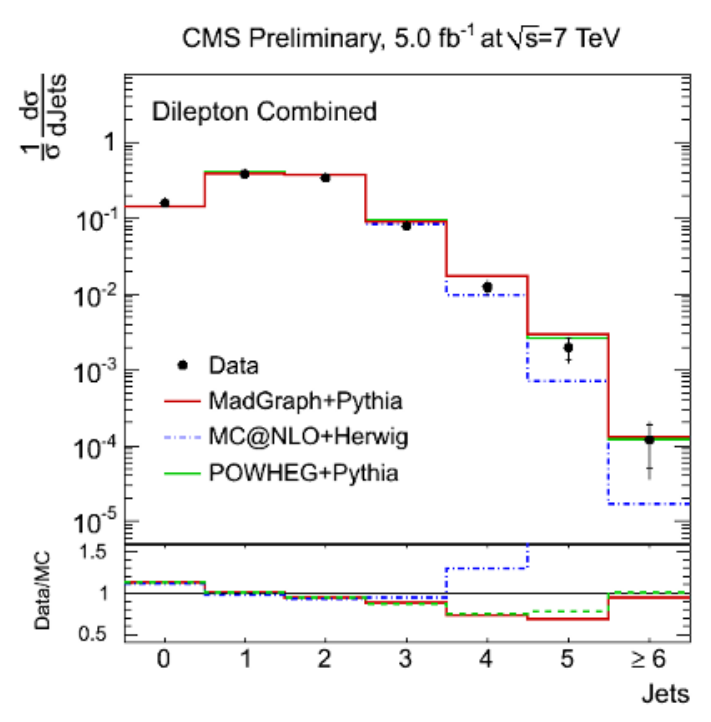

Figure 4. Cross section as a function of the number of jets in top quark pair events compared to different Monte Carlo generator predictions.

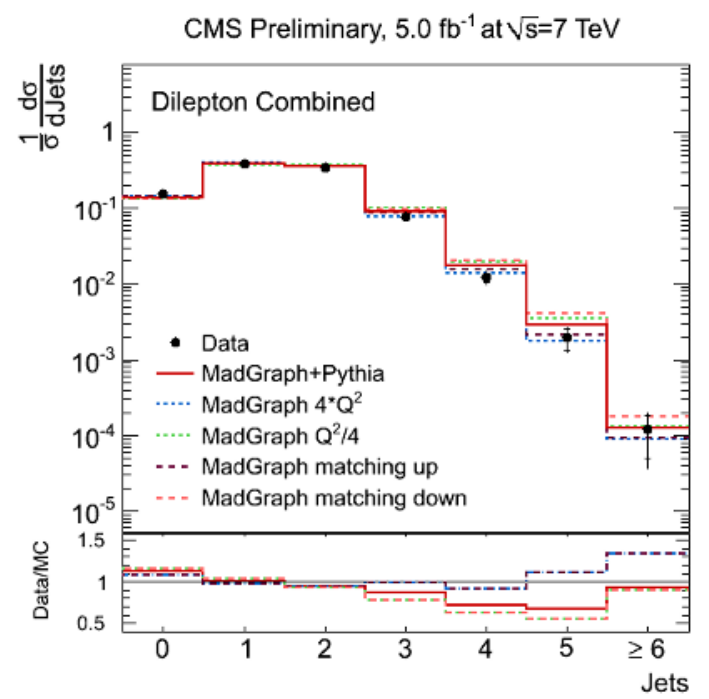

Figure 5. Cross section as a function of the number of jets in top quark pair events within the theoretical uncertainty on factorisation scale and jet-parton matching.

$t \bar{t} b \bar{b}$. A precise measurement of the production cross section of this process helps in the future measurement of the Higgs boson to top quark coupling. In the presented measurement [14] the cross section ratio of the top quark pair production in association with two $b$-jets and in association with two light flavoured jets ( $u-, d-, s_{-}, c$-quarks) is performed in the di-leptonic channels with electrons and muons on $7 \mathrm{TeV}$ collision data. The cross section ratio reduces the overall systematic uncertainty on the measurement due to cancellations of luminosity, jet and lepton efficiency uncertainties. A fit to the $b$-tagged jet multiplicity distribution is performed and the dominant systematic uncertainties are the uncertainty due to the $b$-tag scale factor 
and the factorisation scale uncertainty. The measurement yields a cross section ratio of

$$
\sigma(t \bar{t} b \bar{b}) / \sigma(t \bar{t} j j)=3.6 \pm 1.1 \text { (stat.) } \pm 0.9 \text { (sys.) } \% .
$$

\section{Charge Asymmetry}

At the Tevatron accelerator a forward-backward charge asymmetry in top quark pair events is observed due to the asymmetric initial state of protons and anti-protons which is reflected in the pseudorapidity of the produced top and anti-top quark. At the LHC this effect is not observed due to the symmetric initial state of proton-proton collisions. Nevertheless, at higher orders of perturbation theory the interference of the born and box diagram results in a broader production spectrum of the top quark compared to the anti-top quark in terms of the absolute value of the rapidity distribution.

The rapidity difference of the top and the anti-top quark is defined as

$$
\Delta|y|=\left|y_{t}\right|-\left|y_{\bar{t}}\right| .
$$

Based on this definition the charge asymmetry is defined as

$$
A_{c}=\frac{N^{+}-N^{-}}{N^{+}+N^{-}}
$$

where $N^{+}$is the number of events with $\Delta|y|>0$ and $N^{-}$is the number of events with $\Delta|y|<0$.

\subsection{Measurement of the Charge Asymmetry}

The charge asymmetry is measured in the semileptonic [15] as well as in the di-leptonic channels [17] with electrons and muons at a centre-of-mass energy of $\sqrt{s}=7 \mathrm{TeV}$. The measurement is performed inclusively and as a function of the absolute value of the pseudorapidity, the transverse momentum and the invariant mass of the $t \bar{t}$ system.

In the semi-leptonic channels an inclusive charge asymmetry value of

$$
A_{c}=0.004 \pm 0.010 \text { (stat.) } \pm 0.011 \text { (syst.) }
$$

is measured. The result is compatible with zero and also with the Standard Model expectation [16] of $A_{c}^{\text {Standard Model }}=0.0115 \pm 0.0006$. Differential measurements of the charge asymmetry as a function of the invariant mass and the transverse momentum of the $t \bar{t}$ system are presented in Figures 6 and 7. The dominant systematic uncertainties are the uncertainty on the lepton efficiency determination, the background prediction and the jet energy scale.

All results are compatible with the Standard Model prediction but a reduction of the uncertainties is necessary to draw final conclusions.

The measurement in the di-leptonic channels [17] yields comparable results with larger uncertainties. The inclusive charge asymmetry is measured as

$$
A_{c}=0.050 \pm 0.043 \text { (stat.) }+0.010 /-0.039 \text { (syst.). }
$$

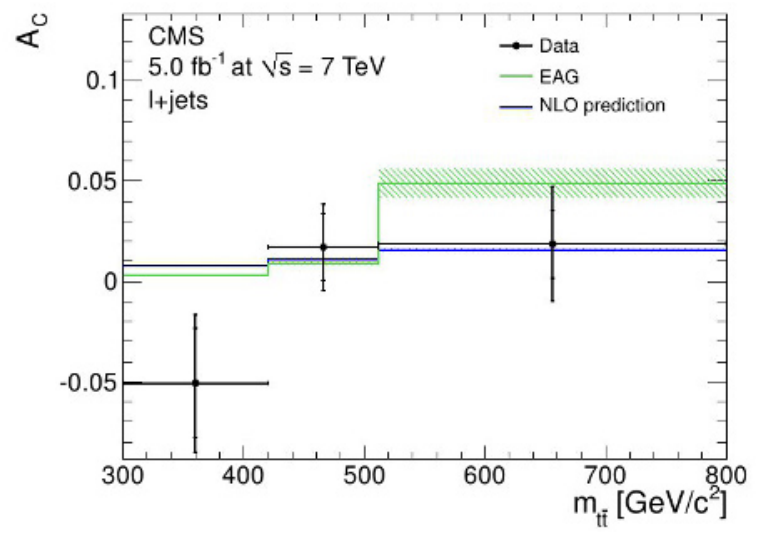

Figure 6. Charge asymmetry as a function of the invariant mass of the $t \bar{t}$ system.

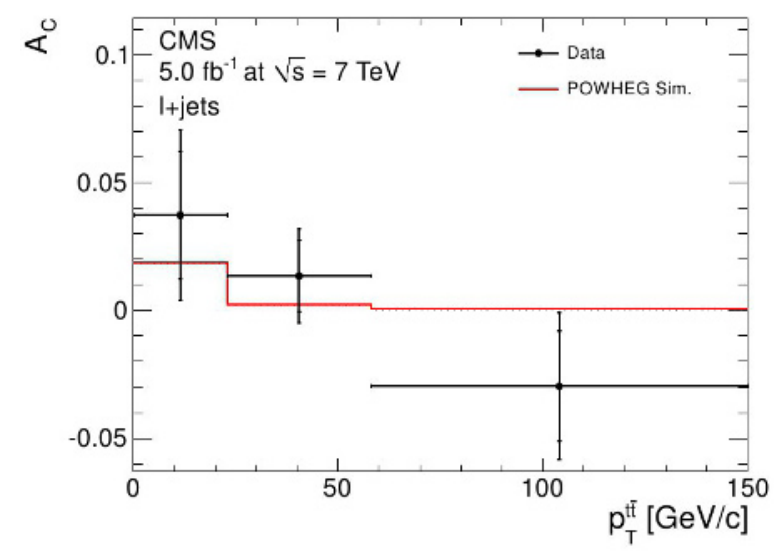

Figure 7. Charge asymmetry as a function of the transverse momentum of the $t \bar{t}$ system.

Again, the measurement does not allow to discriminate between zero and the Standard Model prediction. With the current level of precision it can already be concluded that the data disfavours large deviations from the Standard Model prediction.

\subsection{Leptonic Charge Asymmetry and Top Forward-Backward Asymmetry}

In the di-leptonic channels additional asymmetries are studied [17]. The lepton charge asymmetry is defined as

$$
A_{\text {lepC }}=\frac{N\left(\left|\eta_{\ell^{+}}\right|>\left|\eta_{\ell^{-}}\right|\right)-N\left(\left|\eta_{\ell^{+}}\right|<\left|\eta_{\ell^{-}}\right|\right)}{N\left(\left|\eta_{\ell^{+}}\right|>\left|\eta_{\ell^{-}}\right|\right)+N\left(\left|\eta_{\ell^{+}}\right|<\left|\eta_{\ell^{-}}\right|\right)},
$$

and the forward-backward asymmetry as

$$
A_{\mathrm{top} \mathrm{FB}}=\frac{N\left(\cos \left(\theta_{t}\right)>0\right)-N\left(\cos \left(\theta_{t}\right)<0\right)}{N\left(\cos \left(\theta_{t}\right)>0\right)+N\left(\cos \left(\theta_{t}\right)<0\right)},
$$

where $\theta_{t}$ is the production angle of the top quark in the $t \bar{t}$ rest frame with respect to the direction of the boost of the $t \bar{t}$ system.

The results of the measurement are listed in Table 1. Within the uncertainties all measurements are compatible with the Standard Model predictions. 
Table 1. Results of the measurement of the leptonic charge asymmetry and forward-backward asymmetry in the di-leptonic channels.

\begin{tabular}{lcc}
\hline Unfolded asymmetries & Data & POWHEG \\
\hline$A_{\text {lep C }}$ & $0.010 \pm 0.015 \pm 0.006$ & $0.004 \pm 0.0004$ \\
$A_{\text {top FB }}($ incl. $)$ & $0.011 \pm 0.034 \pm 0.026$ & $0.005 \pm 0.0004$ \\
\hline$A_{\text {top FB }}\left(M_{t \bar{t}}<450 \mathrm{GeV}\right)$ & $0.015 \pm 0.033 \pm 0.034$ & $0.003 \pm 0.0001$ \\
$A_{\text {top FB }}\left(450 \leq M_{t \bar{t}}<550 \mathrm{GeV}\right)$ & $-0.009 \pm 0.050 \pm 0.055$ & $0.005 \pm 0.0001$ \\
$A_{\text {top FB }}\left(M_{t \bar{t}} \geq 550 \mathrm{GeV}\right)$ & $-0.063 \pm 0.071 \pm 0.081$ & $0.007 \pm 0.0001$ \\
\hline
\end{tabular}

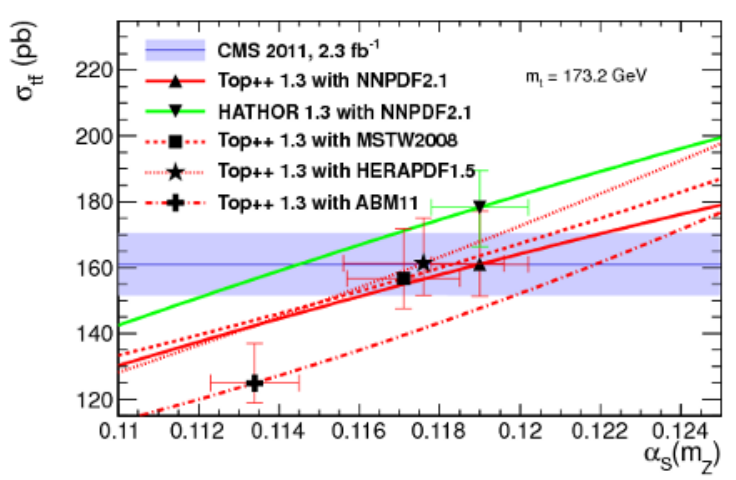

Figure 8. Value of strong coupling $\alpha_{s}$ as a function of the measured top quark production cross section at $\sqrt{s}=7 \mathrm{TeV}$.

\section{Extraction of the Strong Coupling $\alpha_{s}$ from the Cross Section}

The strong coupling constant $\alpha_{s}$ is extracted [18] using a precision measurement of the top quark pair cross section [2] at a centre-of-mass energy of $\sqrt{s}=7 \mathrm{TeV}$ and quoted at the value of the mass of the $Z$ boson. The top quark mass is fixed to its world average value of $173.5 \pm 1.0 \mathrm{GeV}$. The cross section is parametrised as a function of the value of $\alpha_{s}$ using various parton distribution function libraries. A joined likelihood approach is used to extract the value of $\alpha_{s}$. Figure 8 gives an overview of the extracted value of $\alpha_{s}$ for the considered p.d.f. sets. The extracted value with NNPDF2.1 is quoted as final result due to the comparatively low amount of theoretical assumptions in this p.d.f. set. The measurement yields

$$
\alpha_{s}=0.1178+0.0046 /-0.0040
$$

and is compatible with and provides a comparable performance as the current most precise measurements of $\alpha_{s}$ at hadron colliders.

\section{Conclusions}

Top quark pair production is an important means for performing precision tests of the Standard Model. Inclusive and differential cross section measurements have been performed at a centre-of-mass energy of $\sqrt{s}=7 \mathrm{TeV}$ and inclusive measurements are already available for $\sqrt{s}=$ $8 \mathrm{TeV}$. Extensive inclusive and differential studies of the charge asymmetry in top quark events have been performed. A measurement of the strong coupling $\alpha_{s}$ from the top pair production cross section in the di-leptonic channels at $\sqrt{s}=7 \mathrm{TeV}$ yields compatible results to the most precise measurements of $\alpha_{s}$ performed at hadron colliders. All presented measurements are compatible with the Standard Model predictions.

For the future, a transfer of the measurements at a centre-of-mass energy of $\sqrt{s}=7 \mathrm{TeV}$ to the significantly larger dataset at $\sqrt{s}=8 \mathrm{TeV}$ is envisaged. With the increased sensitivity of differential cross section measurements a tuning of the factorisation scale and jet-parton matching parameters in the Monte Carlo simulation will become possible. A larger precision on the measurement of the charge asymmetry will allow to conclusively discriminate between the Standard Model prediction and alternative models.

\section{References}

[1] CMS Collaboration, JINST 3 S08004 (2008)

[2] CMS Collaboration, 1208.2671 [hep-ex] (2011)

[3] J. M. Campbell and R. K. Ellis, 1007.3492 [hep-ph] (2010)

[4] CMS Collaboration, 1212.6682 [hep-ex] (2012)

[5] CMS Collaboration, Phys. Rev. D 85 (2012) 112007 (2012)

[6] CMS Collaboration, CMS PAS TOP-11-004 (2012)

[7] CMS Collaboration, CMS-PAS-TOP-11-007 (2011)

[8] CMS Collaboration, CMS-PAS-TOP-12-006 (2012)

[9] CMS Collaboration, CMS-PAS-TOP-12-007 (2012)

[10] CMS Collaboration, 1211.2220 [hep-ex] (2012)

[11] CMS Collaboration, 1209.4397 [hep-ex] (2012)

[12] CMS Collaboration, CMS-PAS-TOP-12-019 (2012)

[13] CMS Collaboration, CMS-PAS-TOP-12-023 (2012)

[14] CMS Collaboration, CMS-PAS-TOP-12-024 (2012)

[15] CMS Collaboration, Phys. Lett. B717 (2012) 129 (2012)

[16] J. H. Kühn and G. Rodrigo, JHEP 01 (2012) 063 (2012)

[17] CMS Collaboration, CMS-PAS-TOP-12-010 (2012)

[18] CMS Collaboration, CMS-PAS-TOP-12-022 (2012) 